\title{
Optimal diffusive search: nonequilibrium resetting versus equilibrium dynamics
}

\author{
Martin R. Evans ${ }^{(1)}$, Satya N. Majumdar ${ }^{(2)}$ and Kirone Mallick ${ }^{(3)}$ \\ (1) SUPA, School of Physics and Astronomy, University of Edinburgh, Mayfield \\ Road, Edinburgh EH9 3JZ, United Kingdom \\ (2) Univ. Paris-Sud, CNRS, LPTMS, UMR 8626, Orsay F-01405, France \\ (3) Institut de Physique Théorique, CEA Saclay, Gif-Sur-Yvette F-91191, France
}

\begin{abstract}
We study first-passage time problems for a diffusive particle with stochastic resetting with a finite rate $r$. The optimal search time is compared quantitatively with that of an effective equilibrium Langevin process with the same stationary distribution. It is shown that the intermittent, nonequilibrium strategy with non-vanishing resetting rate is more efficient than the equilibrium dynamics. Our results are extended to multiparticle systems where a team of independent searchers, initially uniformly distributed with a given density, looks for a single immobile target. Both the average and the typical survival probability of the target are smaller in the case of nonequilibrium dynamics.
\end{abstract}

PACS numbers: 05.40.-a, 02.50.-r, 87.23.Ge 


\section{Introduction}

Stochastic search problems occur in many fields of science as well as in daily life. The quest for an optimal strategy for locating a target whether inanimate (such as a binding site for a protein at the molecular level [1] or an element in a list) or living (such as a prey for a predator [2]) has been the source of a large number of different algorithms that combine observation, physical mechanisms and computation. Depending on the context, search strategies can be very different, leading to a variety of interesting models (see the special issue [3] devoted to this field of research). One robust class of models called intermittent target search strategies combine phases of slow motion, that allow target detection, and phases of fast motion, during which the searcher relocates but is not reactive (see [4] for a recent review). Such strategies have been observed at different scales: foraging animals, such as humming birds or bumblebees, display intermittent search patterns [5, 6]. The E. Coli bacteria alternates ballistic moves (or 'runs') with random changes of direction ('tumbles') in order to reach regions with high concentration of a chemo-attractant (chemotactic search) [7, 8, 9]. A protein efficiently localizes a specific DNA sequence by alternating $1 \mathrm{~d}$ sliding phases with free $3 \mathrm{~d}$ diffusion ('relocation phases'): this mechanism of 'facilitated diffusion', first proposed by Adam and Delbrück [1], enhances the association rate by two orders of magnitude as compared to the diffusion limit and leads to numerical values that agree quantitatively with experimental results [10, 11] (see [12] for a review).

A simple model of diffusion with stochastic resetting, in which a Brownian particle is stochastically reset to its initial position with a constant rate $r$ was defined and studied in [13]. The stationary state of this process is a non-Gaussian distribution and violates detailed-balance: a non-vanishing steady-state current is directed towards the resetting position. This process can be viewed as an elementary model of an intermittent strategy in which the searcher, having explored its environment unsuccessfully for a while, returns to its initial position and begins a new search. It was also shown in [13] that there exists an optimal resetting rate $r^{*}$ that minimizes the average hitting time to the target. Extensions to space depending rate, resetting to an random position with a given distribution and to a spatial distribution of the target were considered in [14].

The effect of resetting was previously studied in a stochastic multiplicative model of population growth where stochastic resetting events of the population size was shown to lead to a stationary power-law distributed population size distribution [15]. A continuous-time random walk model in the presence of a drift and resetting has also been studied recently [16]. Finally, in the context of search process, a related model has been studied by Gelenbe [17] where searchers are introduced stochastically into the system: there is a single searcher present at a given time with a random lifetime and when the searcher dies, a new searcher is introduced into the system at the initial starting point.

In the mathematics literature, mean first-passage time for a class of random walks with stochastic restarting events has been studied recently from an algorithmic point of 
view [18].

In the present work, we focus on the simple continuous-time Brownian diffusion model with a nonzero resetting rate introduced in [13] and compare quantitatively the reset dynamics with the equilibrium Langevin process that leads to the same stationary distribution. We prove analytically that the non-equilibrium process is more efficient than the equilibrium one by showing that the optimal mean-first passage time is smaller in the former case. We extend our study to the multiparticle problem by considering a team of searchers uniformly distributed on the line and looking for an immobile target. We show that both the average survival probability and the typical survival probability of the non-equilibrium process are smaller than the corresponding quantities for the equilibrium dynamics. This shows that, at least for this model for which exact analytical calculations can be performed, the non-equilibrium strategy systematically defeats the equilibrium behaviour.

The outline of this work is as follows. In section 2, we review the diffusion model with resetting and some of its basic properties, including survival probability and firstpassage time. An effective equilibrium dynamics with the same stationary distribution is defined in section 3 and it is shown that the optimal mean first-passage time associated with that dynamics is always larger than the optimal time if resetting is allowed. In section 4, we investigate the survival probability of a single target in presence of a team of independent searchers. Average and typical survival probabilities display drastically different behaviours (algebraic vs exponential decay with time) both for the nonequilibrium and equilibrium processes. Nevertheless, it is shown that nonequilibrium dynamics is systematically more efficient. The last section is devoted to concluding remarks.

\section{Diffusion with stochastic resetting: nonequilibrium dynamics}

In this section, we recall the definition of the model of diffusion with stochastic resetting and briefly review some basic results, derived in [13].

We consider a single particle on an infinite line starting at the initial position $x_{0}$ at $t=0$. The position of the particle at time $t$ is updated in a small time interval $d t$ by the following stochastic rule [13]:

$$
\begin{aligned}
x(t+d t) & =x_{0} \quad \text { with prob. } r d t \\
& =x(t)+\eta(t) d t \quad \text { with prob. }(1-r d t)
\end{aligned}
$$

where $\eta(t)$ is a Gaussian white noise with mean $\langle\eta(t)\rangle=0$ and the two point correlator $\left\langle\eta(t) \eta\left(t^{\prime}\right)\right\rangle=2 D \delta\left(t-t^{\prime}\right)$. The dynamics thus consists of a stochastic mixture of resetting to the initial position with rate $r$ (long range move) and ordinary diffusion (short range move) with diffusion constant $D$ (see Fig. (1)). Resetting introduces a new length scale $\alpha_{0}^{-1}=\sqrt{D / r}$ in the ordinary diffusion problem.

As shown in [13], the probability density $p(x, t)$ of the particle evolves via the 


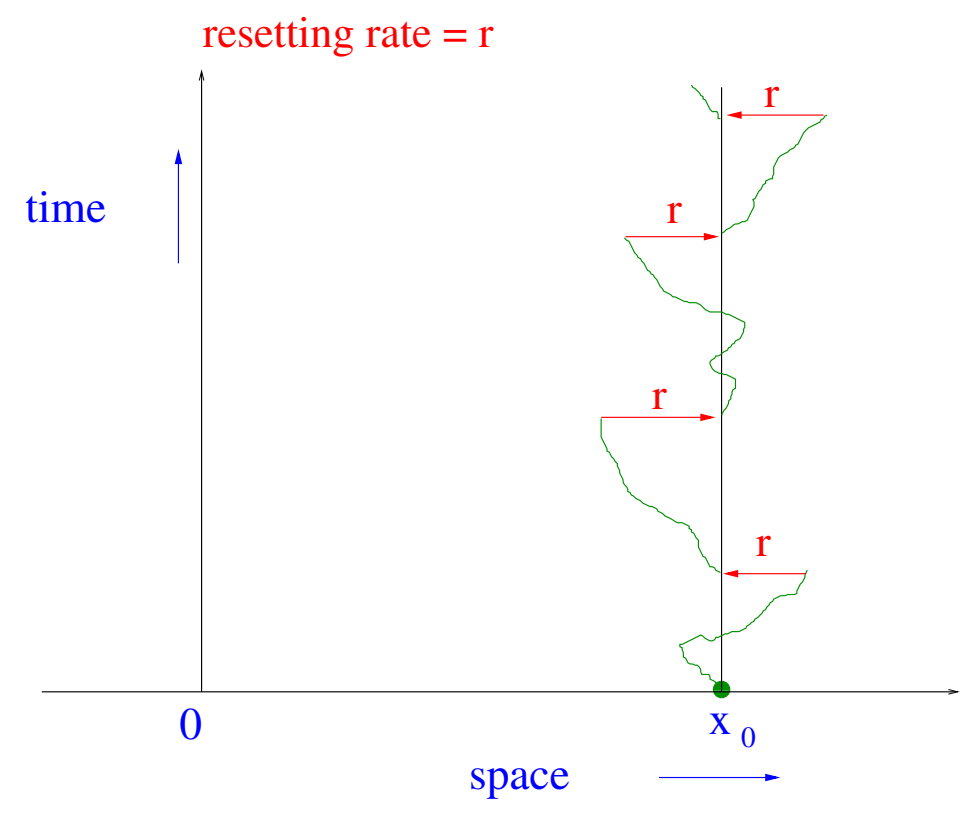

Figure 1. Schematic space-time trajectory of a one dimensional Brownian motion that starts at $x_{0}$ and resets stochastically to its initial position $x_{0}$ at rate $r$.

following Fokker-Planck equation

$$
\partial_{t} p(x, t)=D \partial_{x}^{2} p(x, t)-r p(x, t)+r \delta\left(x-x_{0}\right)
$$

starting from the initial condition $p(x, 0)=\delta\left(x-x_{0}\right)$. We emphasize that the dynamics violates detailed balance manifestly: the current from a position $x$ to $x_{0}$ via the resetting move is not compensated by a current from $x_{0}$ to $x$.

The Fokker-Planck equation (2) admits a stationary solution in the $t \rightarrow \infty$ limit, given by

$$
p_{\mathrm{st}}(x)=\frac{\alpha_{0}}{2} \exp \left[-\alpha_{0}\left|x-x_{0}\right|\right] \quad \text { where } \alpha_{0}=\sqrt{r / D} .
$$

Even though the stationary solution can be expressed as an effective Boltzmann weight: $p_{\text {st }}(x) \propto \exp \left[-V_{\text {eff }}(x)\right]$ with the effective potential $V_{\text {eff }}(x)=\alpha_{0}\left|x-x_{0}\right|$, one should keep in mind that this is actually a nonequilibrium stationary state, and not an equilibrium stationary state. Indeed, in this stationary state detailed balance is violated by a nonzero current in the configuration space. Had the stationary state been an equilibrium one, the current would have exactly vanished.

As shown in [13, 14], the additional resetting parameter $r$ allows us to tune the target search process to make it more efficient. Consider an immobile target at the origin $x=0$ and let the searcher undergo diffusion with resetting dynamics specified in Eq. (1) starting from the initial position $x_{0}>0$. What is the mean first-passage time $T_{\text {reset }}\left(x_{0}, r\right)$ to the origin knowing that the searcher starts at $x_{0}$ ? This average time, $T_{\text {reset }}\left(x_{0}, r\right)$, that the searcher takes to find the target will be taken as a measure of the efficiency of the search process: the smaller the value of $T_{\text {reset }}\left(x_{0}, r\right)$ (for a fixed $\left.x_{0}\right)$, the better is the search. 
In order to calculate $T_{\text {reset }}\left(x_{0}, r\right)$, it is useful to consider the survival probability $q_{\text {reset }}\left(x_{0}, t\right)$ of the target given that the searcher starts at the initial position $x_{0}$ and resets to $x_{0}$. The mean-hitting time is then given quite generally by [20, 21, 22], $T_{\text {reset }}\left(x_{0}, r\right)=\int_{0}^{\infty} t\left(-\partial_{t} q_{\text {reset }}\left(x_{0}, t\right)\right) d t=\int_{0}^{\infty} q_{\text {reset }}\left(x_{0}, t\right) d t$.

More generally, we define $Q\left(x, x_{0}, t\right)$, the survival probability of the target given that the starting position of the searcher is $x$ and its resetting position is $x_{0}$. The initial position $x$ of the searcher can be treated as a variable leading to a backward FokkerPlanck equation for $Q\left(x, x_{0}, t\right)$ [13, 14]. Eventually, at the end of the calculation, one sets $x=x_{0}$ and obtains $q\left(x_{0}, t\right)=Q\left(x=x_{0}, x_{0}, t\right)$. The backward Fokker-Planck equation reads for $x \geq 0$

$$
\partial_{t} Q\left(x, x_{0}, t\right)=D \partial_{x}^{2} Q\left(x, x_{0}, t\right)-r Q\left(x, x_{0}, t\right)+r Q\left(x_{0}, x_{0}, t\right) .
$$

We require the initial condition $Q\left(x, x_{0}, t=0\right)=1$ for all $x>0$ and impose the following boundary conditions: $Q\left(x=0, x_{0}, t\right)=0$ and $Q\left(x \rightarrow \infty, x_{0}, t\right)$ is finite. Equation (4) can be solved explicitly in terms of the Laplace transform $\tilde{Q}\left(x, x_{0}, s\right)=\int_{0}^{\infty} Q\left(x, x_{0}, t\right) e^{-s t} d t$. In particular, setting $x=x_{0}$, we obtain

$$
\tilde{q}_{\text {reset }}\left(x_{0}, s\right)=\tilde{Q}\left(x=x_{0}, x_{0}, s\right)=\frac{1-\exp \left(-\sqrt{\frac{r+s}{D}} x_{0}\right)}{s+r \exp \left(-\sqrt{\frac{r+s}{D}} x_{0}\right)},
$$

thus leading to the mean first-passage time [13]

$$
T_{\text {reset }}\left(x_{0}, r\right)=\int_{0}^{\infty} q\left(x_{0}, t\right) d t=\tilde{q}\left(x_{0}, s=0\right)=\frac{1}{r}\left[\exp \left(\alpha_{0} x_{0}\right)-1\right],
$$

where we recall that $\alpha_{0}=\sqrt{r / D}$. In terms of the dimensionless parameter $\gamma=\alpha_{0} x_{0}=$ $\sqrt{r / D} x_{0}$, we obtain

$$
T_{\text {reset }}\left(x_{0}, \gamma\right)=\left[\frac{e^{\gamma}-1}{\gamma^{2}}\right] \frac{x_{0}^{2}}{D}
$$

We observe that $T_{\text {reset }}\left(x_{0}, \gamma\right)$ diverges in both the limits $\gamma \rightarrow 0$ (pure diffusion) and $\gamma \rightarrow \infty$ (for an infinite resetting rate the particle is localized at $x_{0}$ ). The mean firstpassage time has a unique minimum (see Fig. (2) ) at $\gamma=\gamma_{1}$ where $\gamma_{1}$ is the solution of $\partial_{\gamma} T=0$, i.e., $\gamma_{1}=2\left(1-e^{-\gamma_{1}}\right)$, giving $\gamma_{1}=1.59362 \ldots$ Hence, for fixed values of $D$ and $x_{0}$, there is an optimal resetting rate $r=r_{1}=\gamma_{1}^{2} D / x_{0}^{2}$ that makes the search time minimum and the search process most efficient. The corresponding optimal mean first-passage time is given by

$$
T_{\text {reset }}^{\mathrm{opt}}=\left[\frac{e^{\gamma_{1}}-1}{\gamma_{1}^{2}}\right] \frac{x_{0}^{2}}{D}=1.54414 \ldots \frac{x_{0}^{2}}{D} .
$$

\section{An Effective Equilibrium Dynamics}

We have seen that the resetting with diffusion leads to a current-carrying nonequilibrium stationary state given in Eq. (3). This stationary distribution can be expressed as a 
Boltzmann weight $p_{\mathrm{st}}(x)=\left(\alpha_{0} / 2\right) \exp \left[-V_{\text {eff }}(x)\right]$ with an effective potential

$$
V_{\text {eff }}(x)=\alpha_{0}\left|x-x_{0}\right|
$$

where $\alpha_{0}$ was defined in Eq. (3).

Let us now consider the following Langevin evolution of the particle position with time $t$

$$
\frac{d x}{d t}=-B \partial_{x} V_{\text {eff }}(x)+\eta(t)
$$

where $B$ is the amplitude of the external force and $\eta(t)$ is the same Gaussian white noise as in the previous case, i.e., with $\langle\eta(t)\rangle=0$ and $\left\langle\eta(t) \eta\left(t^{\prime}\right)\right\rangle=2 D \delta\left(t-t^{\prime}\right)$. Note that we have chosen the same diffusion constant $D$ as in the resetting case to reflect the fact that without the reset (in the former case) or without the external potential (in the Langevin case), this equation describes ordinary diffusion with the same diffusion constant $D$ in both cases. The corresponding Fokker-Planck equation for the probability density $P(x, t)$ of the particle is

$$
\partial_{t} P(x, t)=D \partial_{x}^{2} P(x, t)+\partial_{x}\left[B\left(\partial_{x} V_{\text {eff }}(x)\right) P(x, t)\right] .
$$

Eq. (11) can be expressed as a continuity equation, $\partial_{t} P=-\partial_{x} J$ where the current density $J(x, t)=-D \partial_{x} P-B\left(\partial_{x} V_{\text {eff }}(x)\right) P$. The system will then reach a stationary state at long times and if we set the current in the stationary state to be 0 , we arrive at the equilibrium Gibbs-Boltzmann solution which reads, $P_{\text {eq }}(x)=\mathcal{N} \exp \left[-(B / D) V_{\text {eff }}(x)\right]$, where $\mathcal{N}$ is the normalization constant. By choosing $B=D$ and $V_{\text {eff }}(x)=\alpha_{0}\left|x-x_{0}\right|$, we can engineer a zero current equilibrium state which has the same weight as the current carrying nonequilibrium stationary state in the resetting case, i.e.,

$$
P_{\text {eq }}(x)=p_{\text {st }}(x)=\frac{\alpha_{0}}{2} \exp \left[-\alpha_{0}\left|x-x_{0}\right|\right] \quad \text { where } \alpha_{0}=\sqrt{r / D} .
$$

The following natural question then arises. Consider the target search problem, where we have an immobile target at the origin. In the previous section, the searcher was performing normal diffusion with stochastic resetting to its initial position $x_{0}$. Now, suppose that the searcher undergoes instead the Langevin dynamics as in Eq. 10 with the choice $B=D$ and $V_{\text {eff }}(x)=\alpha_{0}\left|x-x_{0}\right|$ that guarantees that both dynamics lead to the same steady state. One is then tempted to compare the efficiency of the nonequilibrium search process with diffusion and reset to the Langevin search process where the searcher's position evolves via Eq. (10). Which process is the more efficient?

To address this question, we need to compute the mean first-passage time $T_{\text {lange }}\left(x_{0}, r\right)$ to the origin of the Langevin process in Eq. (10). With the choice $B=D$ and $V_{\text {eff }}(x)=\alpha_{0}\left|x-x_{0}\right|$ where $\alpha_{0}=\sqrt{r / D}$, the Langevin equation reads

$$
\frac{d x}{d t}=-\alpha_{0} D \operatorname{sgn}\left(x-x_{0}\right)+\eta(t)
$$

where $\operatorname{sgn}(z)$ denotes the sign of $z$. We define $Q_{\text {lange }}\left(x, x_{0}, t\right)$, the probability that the searcher, starting at the initial position $x \geq 0$ and evolving via Eq. (13), does not 
reach the origin (the target) up to time $t$. Equivalently, $Q_{\text {lange }}\left(x, x_{0}, t\right)$ is the survival probability of the target up to time $t$ under the Langevin dynamics of the searcher. Treating the initial position $x$ as a variable, $Q_{\text {lange }}\left(x, x_{0}, t\right)$ satisfies a backward FokkerPlanck equation [20, 21]

$$
\partial_{t} Q_{\text {lange }}\left(x, x_{0}, t\right)=D \partial_{x}^{2} Q_{\text {lange }}\left(x, x_{0}, t\right)-\alpha_{0} D \operatorname{sgn}\left(x-x_{0}\right) \partial_{x} Q_{\text {lange }}\left(x, x_{0}, t\right)
$$

which holds for all $x \geq 0$ with the initial condition $Q_{\text {lange }}\left(x, x_{0}, t=0\right)=1$ for all $x>0$ and the boundary conditions: (i) $Q_{\text {lange }}\left(x=0, x_{0}, t\right)=0$ for all $t$ (absorbing boundary at the origin) and (ii) $Q_{\text {lange }}\left(x \rightarrow \infty, x_{0}, t\right)=1$.

To solve Eq. (14), we consider the Laplace transform $\tilde{Q}_{\text {lange }}\left(x, x_{0}, s\right)=$ $\int_{0}^{\infty} Q_{\text {lange }}\left(x, x_{0}, t\right) e^{-s t} d t$, which, taking into account the initial condition, satisfies

$$
-1+s \tilde{Q}_{\text {lange }}\left(x, x_{0}, s\right)=D \frac{d^{2} \tilde{Q}_{\text {lange }}}{d x^{2}}-\alpha_{0} D \operatorname{sgn}\left(x-x_{0}\right) \frac{d \tilde{Q}_{\text {lange }}}{d x}
$$

Making a shift $\tilde{Q}_{\text {lange }}\left(x, x_{0}, s\right)=1 / s+\tilde{F}\left(x, x_{0}, s\right)$, one finds a homogeneous differential equation for $\tilde{F}$ in $x \geq 0$

$$
D \frac{d^{2} \tilde{F}}{d x^{2}}-\alpha_{0} D \operatorname{sgn}\left(x-x_{0}\right) \frac{d \tilde{F}}{d x}-s \tilde{F}=0
$$

with the boundary conditions: (i) $\tilde{F}\left(x=0, x_{0}, s\right)=-1 / s$ and (ii) $\tilde{F}\left(x \rightarrow \infty, x_{0}, s\right)=0$.

Taking the boundary condition (ii) into account, we obtain

$$
\begin{aligned}
& \tilde{F}\left(x, x_{0}, s\right)=A_{1} \exp \left[-\mu_{2}\left(x-x_{0}\right)\right] \quad \text { for } x>x_{0} \\
& \tilde{F}\left(x, x_{0}, s\right)=B_{1} \exp \left[-\mu_{1}\left(x-x_{0}\right)\right]+B_{2} \exp \left[\mu_{2}\left(x-x_{0}\right)\right] \quad \text { for } x<x_{0}
\end{aligned}
$$

where

$$
\mu_{1}=\left(\sqrt{\alpha_{0}^{2}+4 s / D}+\alpha_{0}\right) / 2, \text { and } \mu_{2}=\left(\sqrt{\alpha_{0}^{2}+4 s / D}-\alpha_{0}\right) / 2 .
$$

From the boundary condition (i) at $x=0$ and using that $\tilde{F}\left(x, x_{0}, s\right)$ and its first derivative $\partial_{x} \tilde{F}\left(x, x_{0}, s\right)$ are continuous at $x=x_{0}$, the three unknown constants $A_{1}$, $B_{1}$ and $B_{2}$ are determined

$$
B_{1}=\frac{2 \mu_{2}}{\mu_{1}+\mu_{2}} A_{1} ; \quad B_{2}=\frac{\mu_{1}-\mu_{2}}{\mu_{1}+\mu_{2}} A_{1} ; \quad \text { and } A_{1}=-\frac{1}{s} \frac{\left(\mu_{1}+\mu_{2}\right)}{\left[2 \mu_{2} e^{\mu_{1} x_{0}}+\left(\mu_{1}-\mu_{2}\right) e^{-\mu_{2} x_{0}}\right]} .
$$

Finally, inserting $x=x_{0}$ leads to

$$
\tilde{q}_{\text {lange }}\left(x_{0}, s\right)=\tilde{Q}_{\text {lange }}\left(x=x_{0}, x_{0}, s\right)=\frac{1}{s}\left[1-\frac{\left(\mu_{1}+\mu_{2}\right)}{\left[2 \mu_{2} e^{\mu_{1} x_{0}}+\left(\mu_{1}-\mu_{2}\right) e^{-\mu_{2} x_{0}}\right]}\right] .
$$

The mean first-passage time is then given by

$$
T_{\text {lange }}\left(x_{0}, r\right)=\int_{0}^{\infty} t\left(-\partial_{t} q_{\text {lange }}\left(x_{0}, t\right)\right) d t=\int_{0}^{\infty} q_{\text {lange }}\left(x_{0}, t\right) d t=\tilde{q}_{\text {lange }}\left(x_{0}, s=0\right) .
$$


Taking the $s \rightarrow 0$ limit in Eq. (21) leads us to

$$
T_{\text {lange }}\left(x_{0}, r\right)=\frac{1}{\alpha_{0}^{2} D}\left[2\left(e^{\alpha_{0} x_{0}}-1\right)-\alpha_{0} x_{0}\right]=\left[\frac{2\left(e^{\gamma}-1\right)-\gamma}{\gamma^{2}}\right] \frac{x_{0}^{2}}{D}
$$

where $\gamma=\alpha_{0} x_{0}=\sqrt{r / D} x_{0}$ is the same dimensionless parameter as defined above (see Eq. (7)). We can now compare the result in Eq. (22) with that of the resetting case, Eq. (7). Using the fact that $e^{\gamma}-1 \geq \gamma$, we see that $T_{\text {lange }}\left(x_{0}, r\right) \geq T_{\text {reset }}\left(x_{0}, r\right)$ for fixed values of $x_{0}$ and $D$.

The minimum value of $T_{\text {lange }}\left(x_{0}, r\right)$ is obtained (see Fig. (2r)) for $\gamma=\gamma_{2}$ where $\gamma_{2}$ is given by the solution of $\partial_{\gamma} T_{\text {lange }}\left(x_{0}, r\right)=0$, i.e., it is the positive root of the equation $2\left(\gamma_{2}-2\right) e^{\gamma_{2}}+\gamma_{2}+4=0$, leading to $\gamma_{2}=1.24468 \ldots$. Thus the optimal mean first-passage time with Langevin dynamics of the searcher is given by

$$
T_{\text {lange }}^{\mathrm{opt}}=\left[\frac{2\left(e^{\gamma_{2}}-1\right)-\gamma_{2}}{\gamma_{2}^{2}}\right] \frac{x_{0}^{2}}{D}=2.38762 \ldots \frac{x_{0}^{2}}{D} .
$$

Comparing with the corresponding result in Eq. (8) for the reset dynamics, we conclude that

$$
\frac{T_{\text {reset }}^{\mathrm{opt}}}{T_{\text {lange }}^{\mathrm{opt}}}=\frac{1.54414 \ldots}{2.38762 \ldots}=0.646728 \cdots \leq 1 .
$$

This shows that the search process via the nonequilibrium diffusion combined with reset mechanism is significantly more efficient than the equilibrium Langevin dynamics of the searcher although the stationary distributions induced by both dynamics are the same: by this measure the nonequilibrium strategy beats the equilibrium dynamics.

\section{Multiparticle problem: Nonequilibrium vs. Langevin dynamics}

In the multiparticle version of the search process, we have a single immobile target at the origin and a team of searchers which are initially uniformly distributed on the line with uniform density $\rho$ (see [23] for a colorful example). The searchers are independent of each other and the position of each searcher evolves stochastically (identical in law for all searchers) starting at its own initial position. This stochastic process, for the moment, is general. When any of the searchers finds the target, the search process is terminated. Let $P_{s}(t)$ denote the survival probability of the target up to time $t$, i.e, the probability that the target has not been found up to $t$ by any of the searchers. To set the problem, we consider $N$ searchers initially distributed uniformly in a box $[-L / 2, L / 2]$ of size $L$ and will eventually take the limit $N \rightarrow \infty, L \rightarrow \infty$, but keeping the density $\rho=N / L$ fixed. Let $x_{i}$ denote the initial position of the $i$-th searcher. Thus, $x_{i}$ is a random variable uniformly distributed in the box $[-L / 2, L / 2]$. Since the searchers are independent, it follows that for a given set of initial positions of the searchers $\left\{x_{i}\right\}$,

$$
P_{s}(t)=\prod_{i=1}^{N} q\left(x_{i}, t\right)
$$




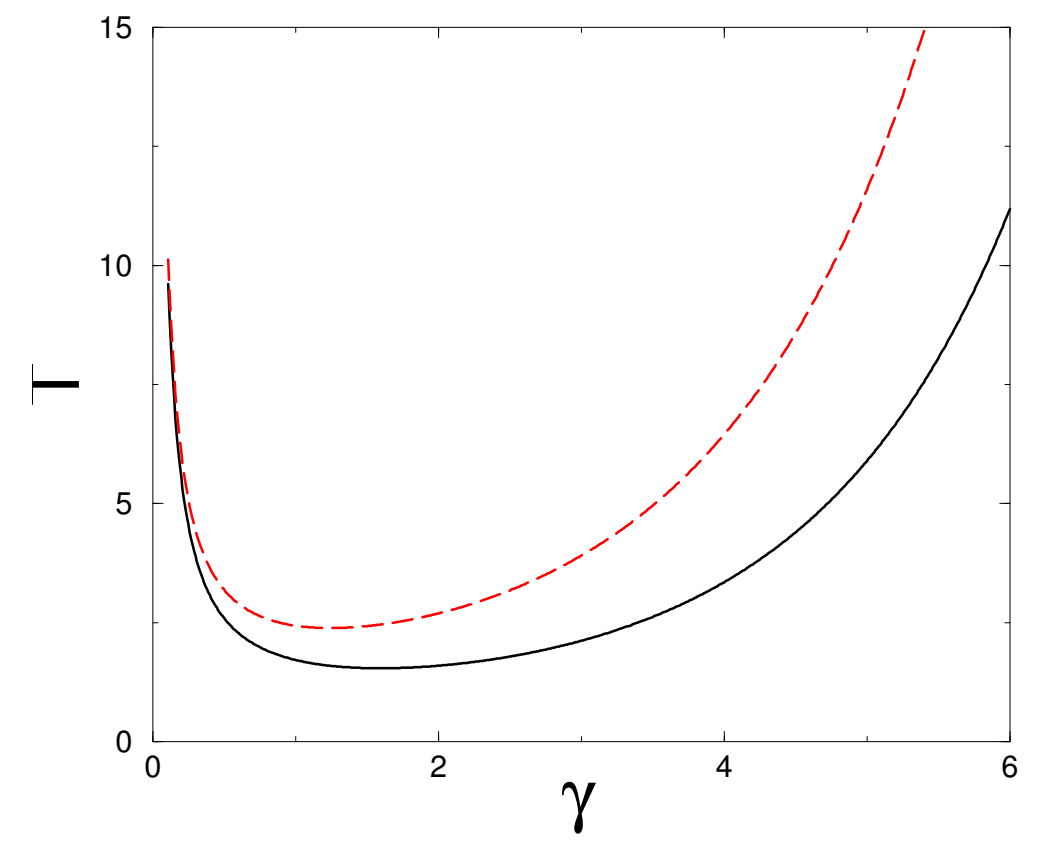

Figure 2. Mean first-passage time $T$ plotted versus the dimensionless parameter $\gamma$ (setting $x_{0}=1$ and $D=1$ ) for the nonequilibrium reset dynamics (shown by the solid (black) line) (see Eq. (7p) and the equilibrium Langevin dynamics (dashed (red) line) (see Eq. (22)). Evidently, the optimal (minimum) $T$ is higher in the equilibrium Langevin case.

where $q\left(x_{i}, t\right)$ is the survival probability of the target due to a single searcher starting at $x_{i}$. For both (i) diffusion with resetting dynamics and (ii) Langevin dynamics in an effective potential $V_{\text {eff }}(x)=\alpha_{0}\left|x-x_{0}\right|$, the Laplace transforms of $q\left(x_{i}, t\right)$ are given respectively in Eqs. (5) and (21). The explicit dependence of $P_{s}(t)$ on $x_{i}$ 's has been suppressed in Eq. 25) for notational convenience.

We first compute the average survival probability (averaged over the initial positions of the searchers) following Ref. [13]. Taking the average of Eq. (25) we obtain

$$
\begin{aligned}
\left\langle P_{s}(t)\right\rangle & =\prod_{i=1}^{N}\left[1-\left\langle\left(1-q\left(x_{i}, t\right)\right\rangle\right]\right. \\
& =\prod_{i=1}^{N}\left[1-\frac{1}{L} \int_{-L / 2}^{L / 2}\left(1-q\left(x_{i}, t\right)\right) d x_{i}\right] \\
& \rightarrow \exp \left[-\rho \int_{-\infty}^{\infty}[1-q(x, t)] d x\right]
\end{aligned}
$$

where in the last step we have exponentiated for large $L$ and then taken the thermodynamic limit $N \rightarrow \infty, L \rightarrow \infty$ but keeping the ratio $\rho=N / L$ fixed. Using further the symmetry $q(x, t)=q(-x, t)$, one finally obtains the general expression of the average survival probability in the multiparticle system

$$
\left\langle P_{s}(t)\right\rangle=\exp \left[-2 \rho \int_{0}^{\infty}[1-q(x, t)] d x\right] .
$$


Actually, this result is rather general (see e.g. [19]) and holds for any stochastic process, the only assumption being that all searchers undergo the same stochastic process. If one can estimate the survival probability $q(x, t)$ of the target at the origin for a single searcher starting at $x$, one has an exact formula for the average survival probability in the multiparticle problem. The quantity $M(t)=\int_{0}^{\infty}[1-q(x, t)] d x$ has the interpretation as the expected maximum up to time $t$ of the single particle process (starting at the origin) and the general formula in Eq. (27) and its discrete-time analogue have been used recently to compute exactly the average survival probability for a number of processes including continuous-time random walks as well as discrete-time Lévy flights [24].

Similarly, the typical survival probability can be estimated [13] by first taking logarithm of Eq. (25), followed by averaging over the initial positions and finally reexponentiating

$$
\begin{aligned}
P_{s}^{\text {typ }}(t) & =\exp \left[\sum_{i=1}^{N}\left\langle\ln \left[q\left(x_{i}, t\right)\right]\right\rangle\right] \\
& \rightarrow \exp \left[2 \rho \int_{0}^{\infty} \ln [q(x, t)] d x\right] .
\end{aligned}
$$

Thus, if we know the survival probability $q(x, t)$ in the single searcher case, we can use the two exact formulae in Eqs. (27) and (28) to estimate respectively the average and the typical survival probability of the target in the multiparticle case.

\subsection{Average survival probability: nonequilibrium vs. equilibrium}

Here, we analyze the asymptotic large $t$ behavior of $\left\langle P_{s}(t)\right\rangle$ in Eq. (27) and compare the expression obtained for the resetting dynamics with the one for the Langevin dynamics.

Let us denote

$$
M(t)=\int_{0}^{\infty}[1-q(x, t)] d x \quad \text { so that }\left\langle P_{s}(t)\right\rangle=\exp [-2 \rho M(t)]
$$

In terms of the Laplace transform of $M(t), \tilde{M}(s)=\int_{0}^{\infty} M(t) e^{-s t} d t$, Eq. 29) reads

$$
\tilde{M}(s)=\int_{0}^{\infty}\left[\frac{1}{s}-\tilde{q}(x, s)\right] d x
$$

We now consider the two cases (i) diffusion with reset and (ii) Langevin dynamics separately.

4.1.1. Nonequilibrium case (diffusion with reset) Substituting $\tilde{q}_{\text {reset }}(x, s)$ from Eq. (5) in Eq. (30) and performing the integration over $x$, one obtains exactly [13]

$$
\tilde{M}(s)=\frac{\sqrt{D(r+s)}}{r s} \ln \left(\frac{r+s}{s}\right) .
$$

The large $t$ behavior of $M(t)$ will be derived from small $s$ behavior of $\tilde{M}(s)$ :

$$
\text { As } s \rightarrow 0, \quad \tilde{M}(s) \approx \sqrt{\frac{D}{r}}\left[-\frac{\ln s}{s}+\frac{\ln (r)}{s}+\ldots\right] .
$$


Inverting the Laplace transform, we find that the leading asymptotic behavior of $M(t)$ for large $t$ is given by

$$
M(t) \approx \sqrt{\frac{D}{r}} \ln t+\sqrt{\frac{D}{r}} \ln (r)+\ldots
$$

Thus, the average survival probability from Eq. (29) decays algebraically for large $t$ [13]

$$
\left\langle P_{s}^{\text {reset }}(t)\right\rangle \approx a_{1} t^{-\theta_{1}} \quad \text { where } \theta_{1}=2 \rho \sqrt{D / r} \quad \text { and } a_{1}=\exp \left[-\theta_{1} \ln r\right]=r^{-\theta_{1}} .
$$

4.1.2. Equilibrium case (Langevin dynamics) Here, we use the expression (21) of $\tilde{q}_{\text {lange }}(x, s)$ in Eq. (30), perform the integration over $x$ exactly and get

$$
\tilde{M}(s)=\left[\frac{\mu_{1}+\mu_{2}}{2 \mu_{1} \mu_{2} s}\right]{ }_{2} F_{1}\left(1, \frac{\mu_{1}}{\mu_{1}+\mu_{2}}, \frac{2 \mu_{1}+\mu_{2}}{\mu_{1}+\mu_{2}}, \frac{\mu_{2}-\mu_{1}}{2 \mu_{2}}\right)
$$

where $\mu_{1,2}$ are defined in Eq. (19), $\alpha_{0}$ in Eq. (3) and the hypergeometric function ${ }_{2} F_{1}(a, b, c, z)$ is given by

${ }_{2} F_{1}(a, b, c, z)=1+\frac{a b}{c} z+\frac{a(a+1) b(b+1)}{c(c+1)} \frac{z^{2}}{2 !}+\frac{a(a+1)(a+2) b(b+1)(b+2)}{c(c+1)(c+2)} \frac{z^{3}}{3 !}+\ldots$

Expanding $\mu_{1,2}$ for small $s$, keeping terms up to $O(s)$ and using $\alpha_{0}^{2} D=r$, one gets

$$
\tilde{M}(s) \approx \frac{\alpha_{0} D}{2 s^{2}}\left[1+\frac{2 s}{r}+\cdots\right]{ }_{2} F_{1}\left[1,1-\frac{s}{r}, 2-\frac{s}{r},-\frac{r}{2 s}-\frac{1}{2}+\frac{s}{2 r}\right]
$$

To make further progress, we use the following identity ${ }_{2} F_{1}(1,1,2,-z)=\ln (1+z) / z$ which follows from the definition Eq. (36). Expanding for small $s$, we find the following leading order behavior of $\tilde{M}(s)$ from Eq. (37)

$$
\tilde{M}(s)=-\frac{1}{\alpha_{0} s} \ln (s)+\frac{1}{\alpha_{0} s} \ln (r / 2)+\ldots
$$

where ... correspond to lower order terms that vanish as $s \rightarrow 0$. This indicates that for large $t$,

$$
M(t) \approx \frac{1}{\alpha_{0}} \ln (t)+\frac{1}{\alpha_{0}} \ln (r / 2)+\ldots
$$

Hence, from Eq. (29), we obtain the average survival probability

$$
\left\langle P_{s}^{\text {lange }}(t)\right\rangle \approx a_{2} t^{-\theta_{2}} \quad \text { where } \theta_{2}=\theta_{1}=2 \rho \sqrt{D / r} \quad \text { and } a_{2}=e^{-\theta_{1} \ln (r / 2)}=(r / 2)^{-\theta_{1}}
$$

Thus the power law exponent $\theta_{2}$ characterizing the algebraic decay of the average survival probability in the Langevin case is identical to the nonequilibrium case, though the amplitude $a_{2}=2^{\theta_{1}} a_{1}$ is larger than $a_{1}$. Hence, for large $t$, the average survival probability in the Langevin case is greater than that of the nonequilibrium case

$$
\left\langle P_{s}^{\text {reset }}(t)\right\rangle \leq\left\langle P_{s}^{\text {lange }}(t)\right\rangle .
$$

Thus, we conclude that on average the target is found faster in the nonequilibrium case than in the equilibrium one. 


\subsection{Typical survival probability: nonequilibrium vs. equilibrium}

We now analyze the asymptotic large $t$ behavior of the typical survival probability, defined in Eq. (28). We define

$$
W(t)=\int_{0}^{\infty} \ln [q(x, t)] d x \quad \text { so that } P_{s}^{\mathrm{typ}}(t)=\exp [2 \rho W(t)] .
$$

We recall that the Laplace transform of $q(x, t)$ is denoted by $\tilde{q}(x, s)$ and its expressions are given in Eqs. (5) and (21) respectively for (i) the diffusion with reset dynamics and (ii) the equilibrium Langevin dynamics in the effective potential $V_{\text {eff }}(x)=\alpha_{0}\left|x-x_{0}\right|$. The two cases will be considered separately.

4.2.1. Nonequilibrium case (diffusion with reset) To analyze the asymptotic large $t$ behavior of $W(t)$, we need to know how $q(x, t)$ behaves for large $t$. The Laplace transform of $q(x, t)$, given in Eq. (5), has a pole at $s=s_{1}$ (for fixed $r$ and fixed $x$ ) which satisfies

$$
s_{1}+r \exp \left[-\sqrt{\frac{r+s_{1}}{D}} x\right]=0 .
$$

Clearly $s_{1}=s_{1}(x)$ depends implicitly on $x$ and one must have $s_{1}(x)<0$. Then, to leading order for large $t$, it follows from Laplace inversion that

$$
q(x, t) \sim \exp \left[s_{1}(x) t\right]=\exp \left[-\left|s_{1}(x)\right| t\right] .
$$

Consequently, from Eq. (42), we find to leading order for large $t$

$$
W(t) \approx-\left[\int_{0}^{\infty}\left|s_{1}(x)\right| d x\right] t .
$$

Hence, the typical survival probability decays exponentially for large $t$ as

$$
P_{s}^{\text {typ }}(t) \sim \exp \left[-2 \rho \kappa_{1} t\right] \quad \text { where } \kappa_{1}=\int_{0}^{\infty}\left|s_{1}(x)\right| d x
$$

To compute $s_{1}(x)$, it is useful to first define $s_{1}(x)=-r(1-u)$, so that Eq. (43) reads, in terms of $u$ and the dimensionless length $z=\alpha_{0} x$

$$
u-1+\exp [-\sqrt{u} z]=0 .
$$

Clearly, as $z \rightarrow \infty, u(z) \rightarrow 1$ and as $z \rightarrow 0, u(z) \rightarrow 0$. Hence

$$
\kappa_{1}=\int_{0}^{\infty}\left|s_{1}(x)\right| d x=\frac{r}{\alpha_{0}} \int_{0}^{\infty}[1-u(z)] d z .
$$

The idea then is to transform the integral over $z$ to an integral over $u$. We then use $d z=d u /\left|u^{\prime}(z)\right|$ where $u^{\prime}(z)=d u(z) / d z$. The derivative can be easily computed from Eq. (47). Expressing Eq. (47) as $z=-\ln (1-u) / \sqrt{u}$ and taking derivative, we get

$$
\frac{d z}{d u}=\frac{1}{2 u^{3 / 2}} \ln (1-u)+\frac{1}{\sqrt{u}(1-u)} .
$$


Hence this gives, using $\alpha_{0}=\sqrt{r / D}$,

$$
\kappa_{1}=\sqrt{r D} \int_{0}^{\infty}[1-u(z)] d z=\sqrt{r D} \int_{0}^{1}(1-u)\left[\frac{1}{2 u^{3 / 2}} \ln (1-u)+\frac{1}{\sqrt{u}(1-u)}\right] d u
$$

The integral in Eq. (50) can be done explicitly to give [13]

$$
\kappa_{1}=4(1-\ln 2) \sqrt{r D} .
$$

Hence finally we find

$$
P_{s}^{\text {typ }}(t) \sim \exp [-8(1-\ln 2) \rho \sqrt{r D} t]=\exp [-(2.45482 \ldots) \rho \sqrt{r D} t] .
$$

As explained in [13], the fact that the average and the typical survival probabilities have different behaviours in the large time limit is a consequence of the memory of the initial conditions in the diffusion process with resetting.

4.2.2. Equilibrium case (Langevin dynamics) In this case, we proceed in exactly the same way as the nonequilibrium case, except that to evaluate $W(t)=\int_{0}^{\infty} \ln [q(x, t)] d x$ in Eq. (42), we need the expression (21) of the Laplace transform of $q_{\text {lange }}(x, t)$. The function $\tilde{q}_{\text {lange }}(x, s)$ has a pole at $s=s_{2}(x)$ which is a root of the equation (with fixed $r$ and fixed $x$ )

$$
2 \mu_{2}\left(s_{2}\right) \exp \left[\mu_{1}\left(s_{2}\right) x\right]+\left(\mu_{1}\left(s_{2}\right)-\mu_{2}\left(s_{2}\right)\right) \exp \left[-\mu_{2}\left(s_{2}\right) x\right]=0,
$$

where we emphasize that $\mu_{1,2}(s)$ defined in Eq. (19) depend on $s$. Because $\mu_{1}>\mu_{2}$, one must have $\mu_{2}\left(s_{2}\right)<0$ and therefore $s_{2}(x)<0$ to fulfil this equation. Thus, we find $q(x, t) \sim \exp \left[-\left|s_{2}(x)\right| t\right]$ for large $t$ and $W(t) \sim-\left[\int_{0}^{\infty}\left|s_{2}(x)\right| d x\right] t$. Consequently

$$
P_{s}^{\text {typ }}(t)=\exp [2 \rho W(t)] \sim \exp \left[-2 \rho \kappa_{2} t\right]
$$

where

$$
\kappa_{2}=\int_{0}^{\infty}\left|s_{2}(x)\right| d x .
$$

To compute $\kappa_{2}$, we reorganize Eq. (53) slightly (using the explicit expressions of $\mu_{1}$ and $\mu_{2}$ ) and express it in terms of the dimensionless length $z=\alpha_{0} x$ as

$$
\sqrt{1+4 s_{2} / r}-1+\exp \left[-\sqrt{1+4 s_{2} / r} z\right]=0 .
$$

where we have used $\alpha_{0}^{2} D=r$. Let us further define $v=1+4 s_{2} / r$ in terms of which Eq. (56) reads

$$
\sqrt{v}-1+\exp [-\sqrt{v} z]=0
$$

Hence, from Eq. (55) we get

$$
\kappa_{2}=\frac{\sqrt{r D}}{4} \int_{0}^{\infty}[1-v(z)] d z
$$


where $v(z)$ is the solution of Eq. (57). Now the solution of Eq. (57) has two branches: $v=0,0 \leq z<1$ and a branch with $v(z)>0$ for $z \geq 1$. Thus (58) becomes

$$
\kappa_{2}=\frac{\sqrt{r D}}{4}\left[1+\int_{1}^{\infty}[1-v(z)] d z\right]
$$

To compute the integral in (59), we use the same trick as above.

$$
\int_{1}^{\infty}[1-v(z)] d z=\int_{0}^{1}[1-v] \frac{d z(v)}{d v} d v .
$$

where $z(v)=-\ln (1-\sqrt{v}) / \sqrt{v}$, which follows from (57). Here, the integral 60 is easily evaluated by integration by parts

$$
\begin{aligned}
\int_{0}^{1}(1-v) \frac{d z(v)}{d v} d v & =[(1-v) z(v)]_{v=0}^{1}+\int_{0}^{1} z(v) d v \\
& =-1-\int_{0}^{1} \frac{\ln \left(1-v^{1 / 2}\right)}{v^{1 / 2}} d v=1 .
\end{aligned}
$$

Therefore from (59) we have

$$
\kappa_{2}=\frac{\sqrt{r D}}{2} .
$$

Hence finally we obtain

$$
P_{s}^{\mathrm{typ}}(t) \sim \exp [-\rho \sqrt{r D} t] .
$$

Comparing this result to Eq. (52), we find that for the multiparticle case as well, the typical survival probability of the target in the equilibrium Langevin case is larger than the nonequilibrium dynamics of diffusion with resetting

$$
\left.P_{s}^{\text {typ }}(t)\right|_{\text {reset }} \leq\left. P_{s}^{\text {typ }}(t)\right|_{\text {lange }} .
$$

This means that the target is found faster in the nonequilibrium case than the equilibrium Langevin case. In other words, the target search process by multiple searchers, as in the case of a single searcher, is more efficient when the searcher undergoes nonequilibrium diffusion and reset dynamics, rather than the equilibrium Langevin dynamics.

\section{Concluding Remarks}

Equilibrium thermodynamics teaches us that dissipation is reduced when a system remains close to an equilibrium state and that the transformations that affect it are quasistatic and reversible. Such a statement of local optimum can not be taken as a paradigm: it can be advantageous in some circumstances to be driven away from equilibrium, by creating non-vanishing stationary currents that break detailed balance and time-reversal invariance.

Optimal search problems provide us with concrete examples in which nonequilibrium can defeat equilibrium. In the present work, we have undertaken a systematic 
comparison between two related search strategies. We consider a predator who performs an intermittent search by alternating phases of free diffusion and resetting jumps. The invariant distribution corresponding to such a dynamics is a nonequilibrium stationary state in which a non-vanishing probability current is directed towards the resetting position. However, one can readily define a fictitious equilibrium Langevin dynamics leading to the same stationary distribution (but with different local transition rates, leading to a vanishing current in the steady state). For the resetting model, this amounts to defining an effective attractive potential centered on the resetting position. The hitting-times to an immobile target can be exactly calculated in both dynamics (nonequilibrium vs equilibrium) and compared. The same problem can be addressed in the case of a team of independent searchers; here the typical and the average survival probabilities differ. Nevertheless, in all the cases we have studied in one dimension, we have shown that the nonequilibrium strategy is advantageous: the intermittent search is always more efficient than equilibrium diffusion. It would be interesting to see if the same conclusion holds in higher dimensions as well and in more realistic search scenarios where, for example, there is a time penalty for resetting.

[1] G. Adam and M. Delbrück, Reduction of dimensionality in biological diffusion processes, in Structural Chemistry and Molecular Biology, A. Rich and N. Davidson Eds. (W.H. Freeman and Company, San Francisco; London, 1968).

[2] W. J. Bell, Searching behaviour: the behavioural ecology of finding resources, (Chapman and Hall, London 1991).

[3] G. Oshanin, K. Lindenberg, H. S. Wio, and S. F. Burlatsky, Efficient search by optimized intermittent random walks, J. Phys. A: Math. Theor. 42, 434008 (2009).

[4] O. Bénichou, C. Loverdo, M. Moreau, and R. Voituriez, Intermittent search strategies, Rev. Mod. Phys. 83, 81 (2011).

[5] Bartumeus F and Catalan J, Optimal search behavior and classic foraging theory J. Phys. A: Math. Theor. 42434002 (2009)

[6] E. R. Raposo, S. V. Buldyrev, M G E dal Luz, G. M. Viswanathan, and H. E. Stanley, Levy Flights and random searches J. Phys. A: Math. Theor. 42434003 (2009)

[7] Y. Kafri and R. A. da Silveira, Steady-State Chemotaxis in Escherichia Coli, Phys. Rev. Lett. 100, 238101 (2008).

[8] M. Sheinman and Y. Kafri, Effects of intersegmental transfers of target location by proteins, Phys. Biol. 6, 016003 (2009).

[9] J. Tailleur and M. E. Cates, Statistical Mechanics of Interacting Run-and-Tumble Bacteria, Phys. Rev. Lett. 100, 218103 (2008).

[10] A. D. Riggs, S. Bourgeois and M. Cohn, The lac repressor-operator interaction. 3. Kinetic studies, J. Mol. Biol. 53, 401 (1970).

[11] O. G. Berg, R. B. Winter and P. H. Von Hippel, Diffusion-driven mechanisms of protein translocation on nucleic acids. 1. Models and theory, Biochemistry 20, 6929 (1981).

[12] L. Mirny, M. Slutsky, Z. Wunderlich, A. Tafvizi, J. Leith and A. Kosmrlj, How a protein searches for its site on DNA: the mechanism of facilitated diffusion, J. Phys. A: Math. Theor. 42, 434013 (2009).

[13] M. R. Evans and S. N. Majumdar, Diffusion with stochastic resetting, Phys. Rev. Lett. 106, 160601 (2011).

[14] M. R. Evans and S. N. Majumdar, Diffusion with optimal resetting, J. Phys. A: Math. Theor. 44, 435001 (2011). 
[15] S. C. Manrubia and D. H. Zanette, Stochastic multiplicative processes with reset events, Phys. Rev. E 59, 4945 (1999).

[16] M. Montero and J. Villarroel, Monotonous continuous-time random walks with drift and stochastic reset events, Preprint arXiv: 1206.4570

[17] E. Gelenbe, Search in unknown environments, Phys. Rev. E 82, 061112 (2010).

[18] S. Janson and Y. Peres, Hitting times for random walks with restarts, SIAM J. Discrete Math. 26, $537(2012)$

[19] M. Tachiya, Theory of diffusion-controlled reactions: formulation of the bulk reaction rate in terms of the pair probability, Radiat. Phys. Chem. 21, 167 (1983)

[20] S. Karlin and H. E. Taylor, A First Course in Stochastic Processes (Academic Press, San Diego, 1975).

[21] S. Redner, A guide to First-Passage Processes (Cambridge University Press, Cambridge 2001).

[22] P. L. Krapivsky, S. Redner and E. Ben-Naim, A Kinetic View of Statistical Physics (Cambridge University Press, Cambridge 2010).

[23] P. L. Krapivsky and S. Redner, Kinetics of a diffusion capture process: lamb besieged by a pride of lions, J. Phys. A: Math. Gen. 29, 5347 (1996).

[24] J. Franke and S. N. Majumdar, Survival probability of an immobile target surrounded by mobile traps, J. Stat. Mech. P05024 (2012). 